\title{
Spectrum Sensing of OFDM Signals in the Presence of CFO: New Algorithms and Empirical Evaluation Using USRP
}

\author{
Anton Blad, Erik Axell and Erik G. Larsson
}

\section{Linköping University Post Print}

N.B.: When citing this work, cite the original article.

(C)2012 IEEE. Personal use of this material is permitted. However, permission to reprint/republish this material for advertising or promotional purposes or for creating new collective works for resale or redistribution to servers or lists, or to reuse any copyrighted component of this work in other works must be obtained from the IEEE.

Anton Blad, Erik Axell and Erik G. Larsson, Spectrum Sensing of OFDM Signals in the Presence of CFO: New Algorithms and Empirical Evaluation Using USRP, 2012, Proceedings of the 13th IEEE International Workshop on Signal Processing Advances in Wireless Communications (SPAWC). 


\title{
SPECTRUM SENSING OF OFDM SIGNALS IN THE PRESENCE OF CFO: NEW ALGORITHMS AND EMPIRICAL EVALUATION USING USRP
}

\author{
Anton Blad, Erik Axell and Erik G. Larsson \\ Department of Electrical Engineering (ISY), Linköping University, 58183 Linköping, Sweden
}

\begin{abstract}
In this work, we consider spectrum sensing of OFDM signals. We deal with the inevitable problem of a carrier frequency offset, and propose modifications to some state-ofthe-art detectors to cope with that. Moreover, the (modified) detectors are implemented using GNU radio and USRP, and evaluated over a physical radio channel. Measurements show that all of the evaluated detectors perform quite well, and the preferred choice of detector depends on the detection requirements and the radio environment.
\end{abstract}

\section{INTRODUCTION}

Spectrum sensing is one of the most essential parts of cognitive radio, and it has been a huge research topic during the last few years [1]. An erroneous decision will either cause interference to the primary user (missed detection) or degrade the spectrum utilization of the secondary user (false alarm). Therefore it is important to sense the spectrum reliably.

Many of the current technologies for wireless communication, such as DVB-T, LTE, WiFi and WiMAX, use orthogonal frequency division multiplexed (OFDM) signaling. Therefore it is reasonable to assume that cognitive radios must be able to detect OFDM signals. The structure of an OFDM signal with a cyclic prefix (CP) gives some very specific properties of the autocorrelation (cf. [1]). Numerous methods for spectrum sensing of OFDM signals have been proposed recently, for example in [2-5]. All of these methods exploit properties of the autocorrelation of the received signal in different ways. They have been derived based on theoretical system models using more or less ideal assumptions, and the performance of these methods have been evaluated in simulations using channel models with a varying degree of realism $[2,3,6]$.

The detectors need not only to work well in a simplified theoretical model, but they also need to be robust to imperfections that arise in a real world system. Some methods take a few imperfections into account, such as unknown channel gain or noise and signal powers [2-4]. However, none of the

This work was supported in part by the Swedish Research Council (VR), the Swedish Foundation for Strategic Research (SSF) and the ELLIIT. E. Larsson is a Royal Swedish Academy of Sciences (KVA) Research Fellow supported by a grant from the Knut and Alice Wallenberg Foundation. underlying system models can of course capture all imperfections of a real world communication chain. To the authors' best knowledge, these detectors have not been implemented in hardware and evaluated and compared in a true physical radio channel.

The main contributions of this work are that

- we propose some modifications to the original spectrum sensing algorithms of $[2,3]$ to cope with the carrier frequency offset,

- we evaluate the performance of the (modified) algorithms of $[2,3,5]$ for detection of an OFDM signal in a physical radio channel, using GNU radio [7] and USRP [8].

\section{MODEL AND PROBLEM FORMULATION}

The problem of spectrum sensing is to determine whether a signal is transmitted or not. That is, we wish to discriminate between the two hypotheses

$$
\begin{aligned}
& H_{0}: y(n)=w(n), n=0, \ldots, N-1, \\
& H_{1}: y(n)=x(n)+w(n), \quad n=0, \ldots, N-1,
\end{aligned}
$$

where $y(n)$ is the observation at time $n, x(n)$ is the primary user's signal and $w(n)$ is noise.

In this work, we compare the detectors proposed in $[2,5]$, and a detector inspired by [3]. To be able to cope with the carrier frequency offset, that was not considered in the original papers, the detectors are slightly modified as described in Sec. 2.2.

\subsection{Original Detectors}

In what follows, we will briefly present the originally proposed detectors. Suppose that the primary transmitter uses OFDM signaling with a cyclic prefix $(\mathrm{CP})$ of length $N_{\mathrm{c}}$. Let $N_{\mathrm{d}}$ be the size of the IFFT used to generate each OFDM symbol. The choice of $N_{\mathrm{c}}$ and $N_{\mathrm{d}}$ is usually determined by standards (cf. [9]), and can therefore be assumed to be known to the detector. An OFDM signal with a $\mathrm{CP}$ is non-stationary and the autocorrelation function of the signal is non-zero for $N_{\mathrm{c}}$ consecutive samples, owing to the repetition of data in the 


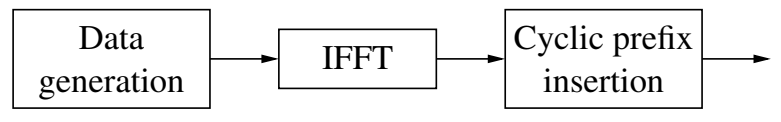

Fig. 1. Baseband data path of OFDM transmitter of primary user

CP. All of the compared methods use estimated autocorrelation in different ways. Let

$$
r(n) \triangleq y(n)^{*} y\left(n+N_{d}\right), \quad n=0, \ldots, N-N_{\mathrm{d}}-1,
$$

and

$R(n) \triangleq \frac{1}{K} \sum_{k=0}^{K-1} r\left(n+k\left(N_{c}+N_{d}\right)\right), n=0, \ldots, N_{c}+N_{d}-1$,

where $K=\left\lfloor\frac{N-N_{\mathrm{d}}}{N_{\mathrm{c}}+N_{\mathrm{d}}}\right\rfloor$ is the number of OFDM symbols.

The original method of [3] uses the real part of the average estimated autocorrelation normalized by the received power, as test statistic. More precisely, the test statistic proposed in [3] is

$$
\frac{\sum_{n=0}^{\left(N-N_{\mathrm{d}}-1\right)} \operatorname{Re}(r(n))}{\sum_{n=0}^{N-1}|y(n)|^{2}}=\frac{K \sum_{n=0}^{\left(N_{\mathrm{c}}+N_{\mathrm{d}}-1\right)} \operatorname{Re}(R(n))}{\sum_{n=0}^{N-1}|y(n)|^{2}} .
$$

Let $\tau \in\left\{0, \ldots, N_{\mathrm{c}}+N_{\mathrm{d}}-1\right\}$ denote the synchronization error, and let $\mathcal{S}_{\tau} \triangleq\left\{\tau, \tau+1, \ldots, \tau+N_{\mathrm{c}}-1\right\}$ $\bmod \left(N_{\mathrm{c}}+N_{\mathrm{d}}\right)$ denote the set of $N_{\mathrm{c}}$ consecutive indices for which the estimated autocorrelation $R(n)$ has non-zero mean, given the synchronization error $\tau$. The original method of [2] uses the test statistic

$$
\max _{\tau} \frac{\sum_{n=0}^{N_{c}+N_{d}-1}|R(n)|^{2}}{\sum_{k \in \mathcal{S}_{\tau}}\left|R(k)-\frac{1}{N_{c}} \sum_{n \in \mathcal{S}_{\tau}} \operatorname{Re}(R(n))\right|^{2}+\sum_{j \notin \mathcal{S}_{\tau}}|R(j)|^{2}} .
$$

The detector of [5] uses a sliding window that sums over $N_{\mathrm{c}}$ consecutive samples, and takes the maximum. The test statistic is

$$
\max _{\tau}\left|\sum_{n=\tau}^{\tau+N_{\mathrm{c}}-1} r(n)\right|
$$

The test statistic (6) takes only one OFDM symbol at a time into account. This was further extended in [2] to utilize multiple symbols. The extended test statistic is then

$$
\max _{\tau}\left|\sum_{n=\tau}^{\tau+N_{\mathrm{c}}-1} R(n)\right| \text {. }
$$

In the sequel, we will refer to (7) as the WRAN detector.

It should be noted that the algorithms based on the test statistics (4) and (5) are constant false-alarm rate (CFAR) detectors by design. That is, no knowledge of SNR or noise power is needed to set the decision threshold. This is not true for the test statistic (7) as will be further discussed in Sec. 3.3.

\subsection{Extensions of Detectors [2] and [3] to CFO}

In the following we explain the proposed modifications of the detectors, that are required to cope with the carrier frequency offset. Most fundamentally, the carrier frequency offset causes the autocorrelation to be complex-valued, and not non-negative real-valued as it was assumed in [2] and [3]. Hence, the estimation of the autocorrelation, made in (5) by summing over the real values of $R(n)$, is not technically sound in the presence of a carrier frequency offset. Therefore, we propose to use the following modified test statistic

$$
\max _{\tau} \frac{\sum_{n=0}^{N_{c}+N_{d}-1}|R(n)|^{2}}{\sum_{k \in \mathcal{S}_{\tau}}\left|R(k)-\frac{1}{N_{c}} \sum_{n \in \mathcal{S}_{\tau}} R(n)\right|^{2}+\sum_{j \notin \mathcal{S}_{\tau}}|R(j)|^{2}},
$$

where the only change is the removal of the real operator in the estimation of the autocorrelation.

The test statistic (4) might become negative in the presence of carrier frequency offset, and will not work at all in general. As a benchmark, inspired by the detector of [3], we will instead use the following test statistic based on the average of the absolute values rather than the real values

$$
\sum_{n=0}^{\left(N_{\mathrm{c}}+N_{\mathrm{d}}-1\right)}|R(n)| .
$$

We will refer to (8) as the GLRT detector since it was derived based on a GLRT-approach, and to (9) as the averaging (avg) detector.

The WRAN detector has been directly implemented according to (7).

\section{HARDWARE AND IMPLEMENTATION}

\subsection{Measurement Setup}

The measurement setup consisted of two laptops, each connected to an Ettus Research USRP1 [8]. One was acting as the primary user and transmitting an OFDM signal, while the other was acting as a secondary user. In the setup, the secondary users are inactive during the sensing of the spectrum. This has several implications for the evaluation of the algorithms, discussed further in Sec. 3.3. The test environment was indoors using the $2.4 \mathrm{GHz}$ ISM band, in a $30 \times 10 \mathrm{~m}$ crowded room with concrete walls, well shielded from interference from other sources such as WLAN and Bluetooth. The antennas were placed at around ten meters in order to allow good SNR estimations, as described in Sec. 3.2. The antennas were not moved during the measurements.

The datapath of the baseband part of the transmitter of the primary user is shown in Fig. 1. First, $N_{\mathrm{d}}$ complex symbols are generated. Of these, the middle $80 \%$, including the DC 


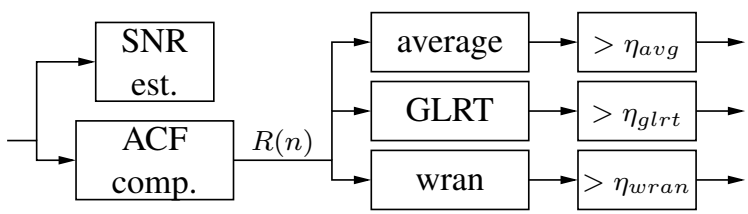

Fig. 2. Baseband data path of spectrum senser

sub-carrier, are randomly generated QPSK symbols, whereas the others are zero. The generated data are IFFT transformed, and then a cyclic prefix of $N_{\mathrm{c}}$ samples is added. The sampling rate of the baseband signal is $8 \mathrm{MS} / \mathrm{s}$. In the USRP1, the baseband signal is upsampled to $128 \mathrm{MS} / \mathrm{s}$, and then modulated to the carrier frequency at $f_{c}=2420 \mathrm{MHz}$.

At the secondary user, the received signal is first downmodulated from $f_{c} \mathrm{MHz}$ to baseband, then sampled at 64 $\mathrm{MS} / \mathrm{s}$. The receiver gain was constant for all the measurements, which simplifies the SNR estimations. Adaptively setting the receiver gain based on the received signal strength can be expected to have an impact on the performance of the algorithms. This is further discussed in Sec. 3.3. However, it is also interesting to compare the performance of the different algorithms when the received noise power is not affected by the receiver gain, and this is the focus of this paper. After sampling, the signal is down-sampled to $8 \mathrm{MS} / \mathrm{s}$ before the baseband processing.

The datapath of the baseband part of the secondary user is shown in Fig. 2. The signal-to-noise ratio of the input is estimated, as described further in Sec. 3.2. Also, the averaged estimation of the time-variant auto-correlation function $R(n)$ is computed. $R(n)$ is then used by the three different sensing algorithms, which each produce metrics for the received data blocks. The metrics are compared to the algorithm dependent thresholds $\eta_{\text {avg }}, \eta_{\text {glrt }}$, and $\eta_{\text {wran }}$ to produce answers to the hypothesis tests (1). In this way, the three algorithms are using the same received data, ensuring a fair comparison.

\subsection{SNR Estimations}

To properly present the detection performance at different SNR levels, the SNR has been estimated from measurements as explained in the following. Note, however, that knowledge of the SNR is not required for any of the detectors, but this is only needed for the purpose of presenting the results. The SNR region of interest for spectrum sensing is low, at around -20 to $-10 \mathrm{~dB}$. Estimating the SNR at these levels is not a simple problem, and therefore the following approach is taken:

1. Measure $P_{\text {noise }}$ as the received signal power with the transmitter off.

2. Measure $P_{f s}$ as the received signal power with the transmitter at full power.

3. Compute the received SNR with the transmitter at full power as $\mathrm{SNR}_{0 d B f s}=10 \log _{10} \frac{P_{f s}-P_{\text {noise }}}{P_{\text {noise }}}$.
4. Determine the SNR at a transmitted signal level of $A$ $\mathrm{dBfs}$ as $\mathrm{SNR}=S N R_{0 d B f s}+A$.

Assuming that the received signal power at full transmitter power is sufficiently large (i.e., the transmitter and receiver are sufficiently close), the full scale SNR estimation $\mathrm{SNR}_{0 d B f s}$ has high accuracy. Additionally ensuring that the full power is still in the transmitter's linear region, the received SNR can be determined reliably from the full scale SNR estimation and the transmitted signal level.

\subsection{Noise Considerations}

The received noise power of the transmitter varies for several reasons. The main source is normally thermal noise contributions from different stages of the receiver. The thermal noise varies with the operating temperature of the circuits. Another noise source is the presence of an interfering transmitter in the same or an adjacent frequency band. In particular, the presence of another active secondary user may constitute a strong interference for a secondary user performing spectrum sensing. The noise sources are then amplified by the receiver, such that the noise power additionally depends on the receiver gain. Finally, filters and amplifiers have different characteristics for different frequencies, such that the noise power also depends on the carrier frequency.

In this paper, care has been taken to keep the received noise power as constant as possible, in order to focus on the performance of the algorithms for different choices of OFDM signal parameters. Therefore, the measurements have been conducted in an environment where the interference has been limited, and the carrier frequency and the gain setting of the receiver has been kept constant.

Regarding the investigated algorithms, the averaging detector and the WRAN detector (as described in Sec. 2.2) are sensitive to variations in the received noise power, whereas the GLRT detector is not. In particular the decision threshold of the averaging and WRAN detectors depend on the noise power in addition to the desired probability of false alarm. This kind of noise dependent detectors generally suffers severely from even small variations of the noise power. This phenomenon is, for example, very well known for the commonly used energy detector (cf. [1]). However, as seen in Sec. 4, the performance of the noise dependent detectors is very good so that none of the algorithms are severely impacted by variations in the noise power.

\section{MEASURED RESULTS}

Throughout all measurements, the probability of false alarm was set to $P_{\mathrm{FA}}=0.05$. For the $\mathrm{SNR}$ curves, the number of false detections for each measurement is at least 100 for the $N_{d}=2048$ measurements in Figs. 3 and 4, and at least 20 for the $N_{d}=256$ measurements in Fig. 6. For the ROC curves, 


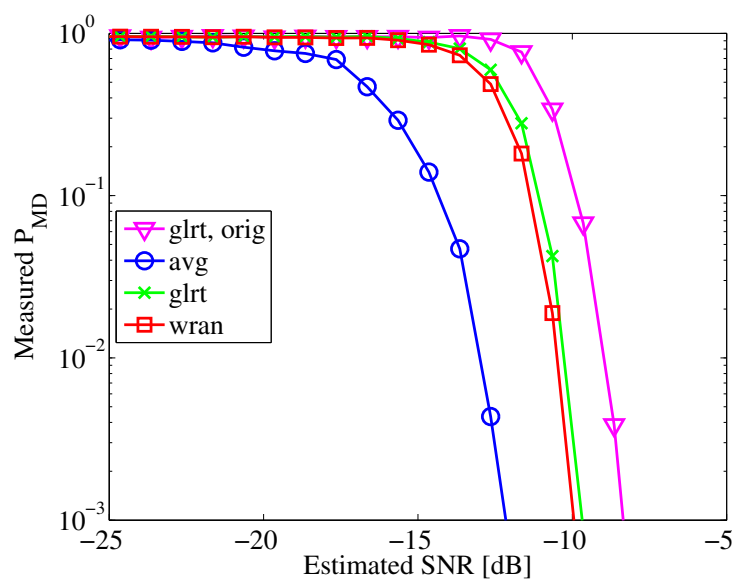

Fig. 3. Probability of missed detection as a function of SNR, with the parameters set to $N_{\mathrm{d}}=2048, N_{\mathrm{c}}=64$ and $K=64$, corresponding to a sensing time of $17 \mathrm{~ms}$.

Fig. $5\left(N_{d}=2048\right)$ is based on 2000 measurements, whereas Fig. $7\left(N_{d}=256\right)$ is based on 10000 measurements.

The SNR estimation was done according to Sec. 3.2, with a transmitted signal at full power corresponding to a received $\mathrm{SNR}$ at $6 \mathrm{~dB}$. Thereafter, the measurements for different received SNR were performed by varying the transmitted signal power. The receiver gain was set such that the quantization noise of the ADC is at $-31 \mathrm{~dB}$ in the figures.

For the SNR curves, the decision thresholds were computed empirically based on noise-only samples (i.e. the transmitter was switched off), to achieve the desired $P_{\mathrm{FA}}$. The calibrations used 20000 and 100000 measurements, for $N_{d}=$ 2048 and $N_{d}=256$, respectively.

The TV broadcast spectra have been thought of as one of the main resources for secondary use, and therefore DVBT2 has been used as a base for choosing the signal parameters. However, since the frequencies are licensed, the measurements have been made in the unlicensed $2.4 \mathrm{GHz}$ ISM band. Since the measure of interest is SNR rather than received signal power, the different propagation characteristics of these frequencies are assumed to affect the results minimally.

In the first results, shown in Figs. 3-5, the parameters of the OFDM signal were chosen to $N_{\mathrm{d}}=2048$ and $N_{\mathrm{c}}=64$. This is one of the transmission modes used in the DVB-T2 standard [9]. Figure 3 shows the probability of missed detection versus SNR for $K=64$, corresponding to a sensing time of $17 \mathrm{~ms}$, and Fig. 4 shows the result for a longer sensing time with $K=1024$, corresponding to a sensing time of $270 \mathrm{~ms}$. It is clear the the GLRT (8) and the WRAN detector (7) have similar performance for both sensing times. However, the performance of these two detectors improves significantly with increasing sensing time, relative to the detector (9). This can be explained by the large variance of the signal contribution to averaged auto-correlation $R(n)$, i.e.,

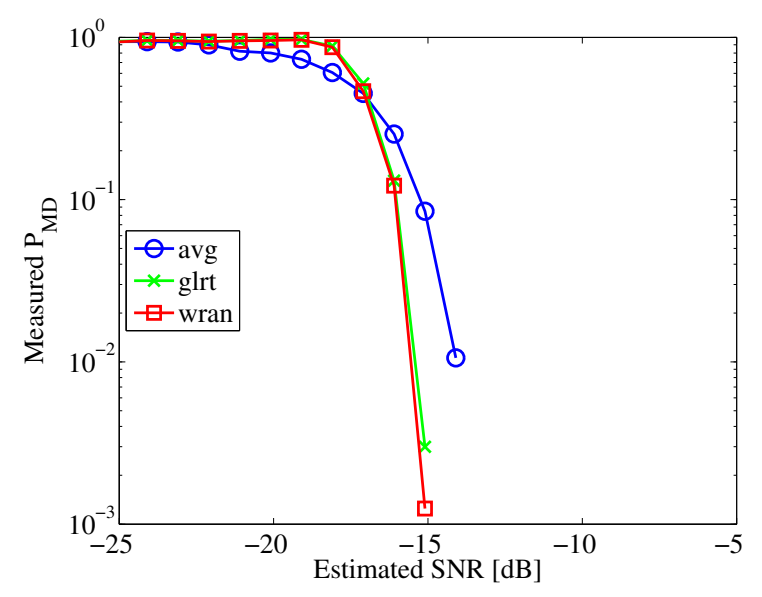

Fig. 4. Probability of missed detection as a function of SNR, with the parameters set to $N_{\mathrm{d}}=2048, N_{\mathrm{c}}=64$ and $K=$ 1024 , corresponding to a sensing time of $270 \mathrm{~ms}$.

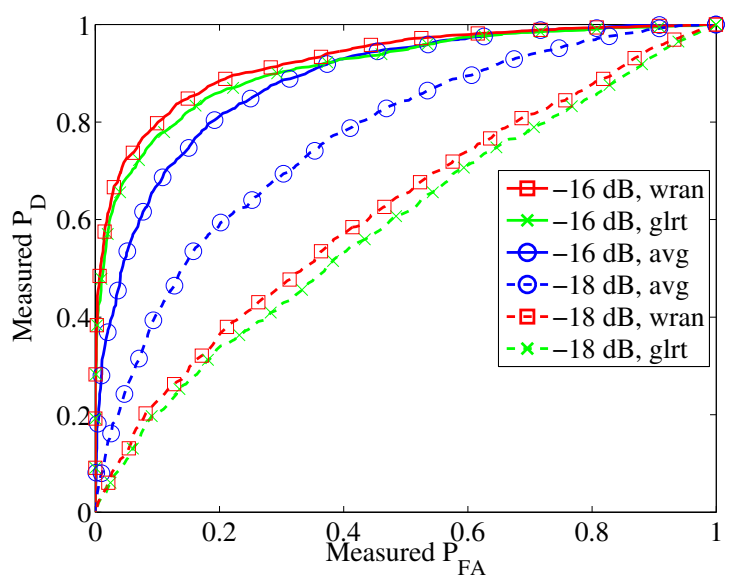

Fig. 5. ROC curve, with the parameters set to $N_{\mathrm{d}}=2048$, $N_{\mathrm{c}}=64$ and $K=1024$, corresponding to a sensing time of $270 \mathrm{~ms}$.

$\mathbb{E}\left\{|R(n)|^{2}\right\}-\mathbb{E}\{|R(n)|\}^{2}$ is large in relation to $\mathbb{E}\{|R(n)|\}$. Therefore, for short sensing times, the performance of the GLRT and WRAN detectors are inhibited by the lack of structure in the transmitted signal. Figure 3 also shows the performance of the original GLRT detector (5), which suffers severely from the inexact estimation of the autocorrelation. Similar results were obtained were obtained for the other scenarios. The original averaging algorithm (4) produced unusable results due to reasons explained in Sec. 2.2, and those results are therefore not shown.

Figure 5 shows the receiver operating characteristics (ROC) curves for the same parameters as in Fig. 4, at SNRs equal to -16 and $-18 \mathrm{~dB}$. The better performance of the averaging detector, compared with the WRAN and GLRT detectors, for low SNR is evident. Similar results were reported in [2].

Figure 6 shows results similar to Figs. 3-4, but for a smaller FFT size $\left(N_{\mathrm{d}}=256\right)$. Similar observations can be 


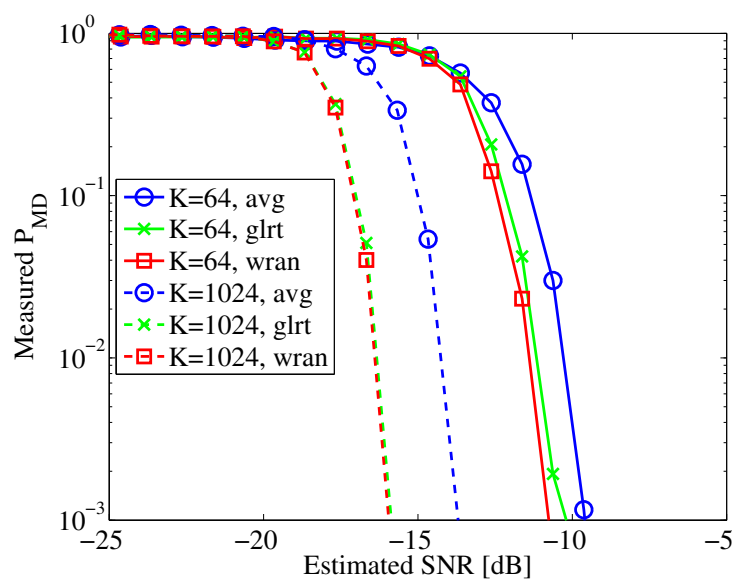

Fig. 6. Probability of missed detection as a function of SNR, with the parameters set to $N_{\mathrm{d}}=256$ and $N_{\mathrm{c}}=64$

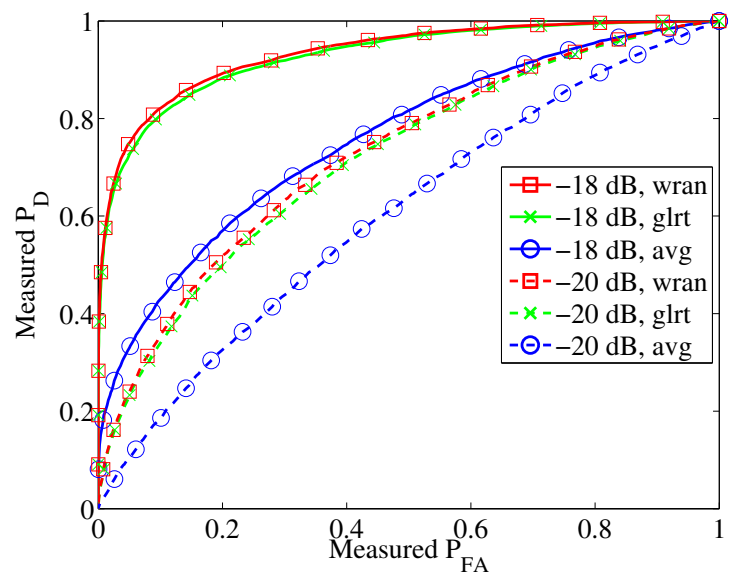

Fig. 7. ROC curve, with the parameters set to $N_{\mathrm{d}}=256$, $N_{\mathrm{c}}=64$ and $K=1024$

made as for the larger FFT size in Figs. 3-4. Worth noting is that the performance of all detectors is improved with a smaller FFT size but a fixed CP size. This is particularly visible for the averaging detector. The reason is that a smaller part $\left(N_{\mathrm{c}} /\left(N_{\mathrm{c}}+N_{\mathrm{d}}\right)\right)$ of each received symbol differs between the two hypotheses for a larger FFT size (and fixed CP length).

Figure 7 shows the ROC curves for the same parameters as in Fig. 6 with $K=1024$, at SNRs equal to -18 and -20 $\mathrm{dB}$. Again, the relative performances of the different detectors are similar to those shown in Fig. 5. However, the WRAN and GLRT detectors outperform the averaging detector more significantly for the smaller FFT size, for the reasons explained.

\section{CONCLUDING REMARKS}

The measurements showed that the modified GLRT detector and the WRAN detector perform best under most of the considered scenarios, but the averaging detector is preferred in some cases. In general, the preferred detector depends on the parameters of the signal, as well as the detection requirements such as detection time, detection probability and SNR.

In this work, the measurement setup was somewhat idealized, since all measurements were conducted in a shielded environment. Future work should also include more realistic scenarios, such as interference from other secondary users or neighboring frequency bands. More practical and better use of varying gain control should also be considered. These factors will cause varying noise power which is a known weakness of, for example, the WRAN detector and demands further investigation.

We showed that all of the detectors, after minor modifications, work reasonably well in a real-world physical radio channel. This is very promising for the future work on spectrum sensing and cognitive radio.

\section{REFERENCES}

[1] E. Axell, G. Leus, E. G. Larsson, and H. V. Poor, "Spectrum sensing for cognitive radio: State-of-the-art and recent advances," IEEE Signal Process. Mag., to appear.

[2] E. Axell and E. G. Larsson, "Optimal and sub-optimal spectrum sensing of OFDM signals in known and unknown noise variance," IEEE J. Sel. Areas Commun., vol. 29, pp. 290-304, Feb 2011.

[3] S. Chaudhari, V. Koivunen, and H. V. Poor, "Autocorrelation-based decentralized sequential detection of OFDM signals in cognitive radios," IEEE Trans. Signal Process., vol. 57, pp. 2690-2700, Jul. 2009.

[4] J. Lundén, V. Koivunen, A. Huttunen, and H. V. Poor, "Collaborative cyclostationary spectrum sensing for cognitive radio systems," IEEE Trans. Signal Process., vol. 57, pp. 4182-4195, Nov. 2009.

[5] Huawei Technologies and UESTC, "Sensing scheme for DVB-T," Nov. 2006, iEEE Std.802.22-06/0263r0. [Online]. Available: https://mentor.ieee.org/802.22/dcn/06/ 22-06-0263-00-0000-huawei-sensing-scheme-for-dvb-t. doc

[6] S. Chaudhari, J. Lundén, and V. Koivunen, "Collaborative autocorrelation-based spectrum sensing of OFDM signals in cognitive radios," in CISS, Princeton, NJ, Mar. 2008, pp. 191-196.

[7] “GNU Radio project home page," Feb. 2012. [Online]. Available: http://www.gnuradio.org/

[8] "Ettus research home page," Feb. 2012. [Online]. Available: http://www.ettus.com/

[9] DVB Project Office, "DVB fact sheet - 2nd generation terrestrial," Aug. 2011. [Online]. Available: http://www. dvb.org/technology/fact_sheets/DVB-T2_Factsheet.pdf 